\title{
Accelerated FRET-PAINT microscopy
}

\author{
Jongjin Lee ${ }^{1}$, Sangjun Park ${ }^{1}$ and Sungchul Hohng ${ }^{1,2^{*}}$ (i)
}

\begin{abstract}
Recent development of FRET-PAINT microscopy significantly improved the imaging speed of DNA-PAINT, the previously reported super-resolution fluorescence microscopy with no photobleaching problem. Here we try to achieve the ultimate speed limit of FRET-PAINT by optimizing the camera speed, dissociation rate of DNA probes, and bleed-through of the donor signal to the acceptor channel, and further increase the imaging speed of FRETPAINT by 8-fold. Super-resolution imaging of COS-7 microtubules shows that high-quality 40-nm resolution images can be obtained in just tens of seconds.
\end{abstract}

Keywords: Super-resolution fluorescence microscopy, Single-molecule localization microscopy, FRET, FRET-PAINT

\section{Introduction}

Different types of super-resolution fluorescence microscopy techniques have been developed to overcome the diffraction limit of optical microscopy [1-7]. The achievement, however, was obtained by sacrificing imaging speed and total observation time; with increased optical resolution, the imaging speed is generally slowed-down and the photobleaching problem of fluorophores becomes exacerbated resulting in the limited total imaging time. DNA-PAINT (Point Accumulation for Imaging in Nanoscale Topography [8]) technique has overcome the photobleaching problem by using transient binding of a fluorescently labeled short DNA strand (imager strand) to a docking DNA strand conjugated to target molecules [9]. The binding rates of DNA probes, however, are notoriously slow, and as a result, DNA-PAINT has an extremely slow imaging speed (1-3 frames per hour), impeding widespread usage of DNA-PAINT in biological imaging. To solve this problem of DNA-PAINT, FRET-PAINT microscopy has been introduced independently by two groups $[10,11]$. In this technique, two short DNA strands labeled with donor and acceptor are used as fluorescence probes. Because only the acceptor signal is used for single-molecule localization, more concentrated DNA probes could be used, resulting in a 30-fold increase in imaging speed compared to DNA-PAINT [10].

\footnotetext{
* Correspondence: shohng@snu.ac.kr

${ }^{1}$ Department of Physics and Astronomy, Seoul National University, Seoul 08826, Republic of Korea

${ }^{2}$ Institute of Applied Physics, Seoul National University, Seoul 08826, Republic of Korea
}

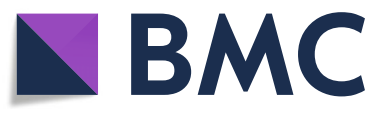

The ultimate speed limit of FRET-PAINT has not been characterized yet. The imaging speed of FRET-PAINT is influenced by the camera speed, dissociation rate of DNA probes, and maximum concentration of DNA probes. In this paper, we optimize the three factors to reach the speed limit of FRET-PAINT imaging, and as a result, report a super-resolution fluorescence microscopy that can provide $40-\mathrm{nm}$ resolution images in tens of seconds. In this process, we recognized the previously uncharacterized photo-induced damage of DNA probes, which currently limits both the imaging speed and the observation time of FRET-PAINT.

\section{Results}

\section{Accelerated dissociation of donor strands}

The experimental scheme of FRET-PAINT and instrumental setup are briefly presented in Fig. 1a. In the previous work, we used an EMCCD (iXon Ultra DU-897 U-CS0-\#BV, Andor) with a maximum frame rate of $56 \mathrm{~Hz}$ and $512 \times 512$ imaging area. Due to slow dissociation of DNA probes, however, actual frame rate used was $10 \mathrm{~Hz}$. In this work, we replaced the EMCCD with an sCMOS camera (ORCA-Flash $4.0 \mathrm{~V} 2$, Hamamatsu) with a maximum frame rate of $400 \mathrm{~Hz}$ for the same size of an imaging area. Due to the photo-induced damage of DNA probes that will be explained later in more detail, however, the maximum frame rate used was $200 \mathrm{~Hz}$. To compensate for short exposure time, illumination intensity should be increased proportionally to frame rate. For the

(C) The Author(s). 2018 Open Access This article is distributed under the terms of the Creative Commons Attribution 4.0 International License (http://creativecommons.org/licenses/by/4.0/), which permits unrestricted use, distribution, and reproduction in any medium, provided you give appropriate credit to the original author(s) and the source, provide a link to the Creative Commons license, and indicate if changes were made. The Creative Commons Public Domain Dedication waiver (http://creativecommons.org/publicdomain/zero/1.0/) applies to the data made available in this article, unless otherwise stated. 


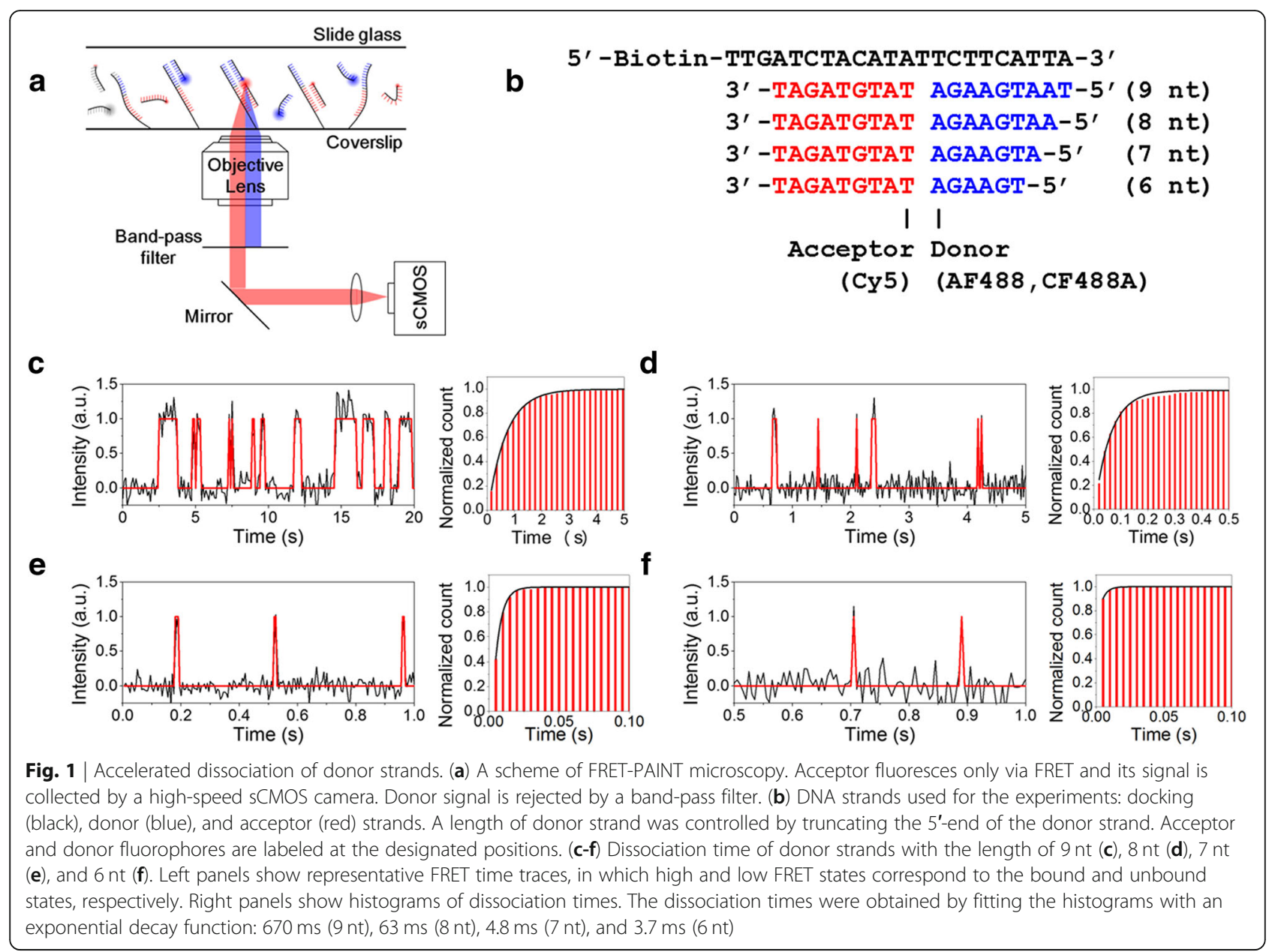

same reason of photo-induced DNA damage, we used an illumination power of $1.5 \mathrm{~kW} / \mathrm{cm}^{2}$, just 3.3 -fold increase from $460 \mathrm{~W} / \mathrm{cm}^{2}$ that was used in the previous work.

To fully utilize the increased frame rate of an sCMOS camera, the switching rate of DNA probes should be increased as well; if the dissociation of DNA probe is slow, single-molecule spots start to overlap at lower probe concentrations, limiting the overall imaging speed. We determined the dissociation times of donor strands with various lengths. Four different donor strands were tested (Fig. 1b, blue). Figure 1c-f show representative time traces (left) and histograms of dissociation time of the donor strands (right). The dissociation times obtained were $670 \mathrm{~ms}(9 \mathrm{nt}), 63 \mathrm{~ms}(8 \mathrm{nt}), 4.8 \mathrm{~ms}(7 \mathrm{nt})$, and $3.7 \mathrm{~ms}$ $(6 \mathrm{nt})$. The dissociation times of $7 \mathrm{nt}$ and $6 \mathrm{nt}$ donor strands were measured to be shorter than the camera exposure time ( $5 \mathrm{~ms})$, and should be considered inaccurate. We selected $7 \mathrm{nt}$ donor strands for the frame rate of 100 or $200 \mathrm{~Hz}$ used in this work. Auer et al. previously used $7 \mathrm{nt}$ donor strands [11], but the dissociation time of the strand was much longer $(88 \mathrm{~ms})$ than ours $(4.8 \mathrm{~ms})$.

\section{Improved signal-to-noise ratio (SNR)}

Background noise coming from floating donor and acceptor strands limits the maximum probe concentration that can be used. To reduce the background noise, and as a result to increase the maximum probe concentrations to give reasonable signal-to-noise ratio (SNR), we first tried different donor-acceptor pairs other than the Alexa Fluor 488 (AF488, Invitrogen)-Cy5 (GE Healthcare) pair used in the previous work. In terms of background noise, the more the spectral separation of donor and acceptor emissions, the better SNR. Absorption and excitation spectra of Alexa dyes [12], Atto dyes [13, 14], CF dyes [15], and Cy dyes [12] were compared, and CF488A (Biotium) and CF660R (Biotium) were selected as candidates to replace AF488 and Cy5, respectively. Figure 2a compares excitation (dashed lines) and emission (solid lines) spectra of AF488 (black), CF488A (red), Cy5 (magenta), and CF660R (violet). The absorption and emission spectra of CF488A is blue-shifted to those of AF488 whereas their extinction coefficients are similar at the peaks. On the other hand, the emission spectrum of CF660R is red-shifted to that of Cy5. As an additional 


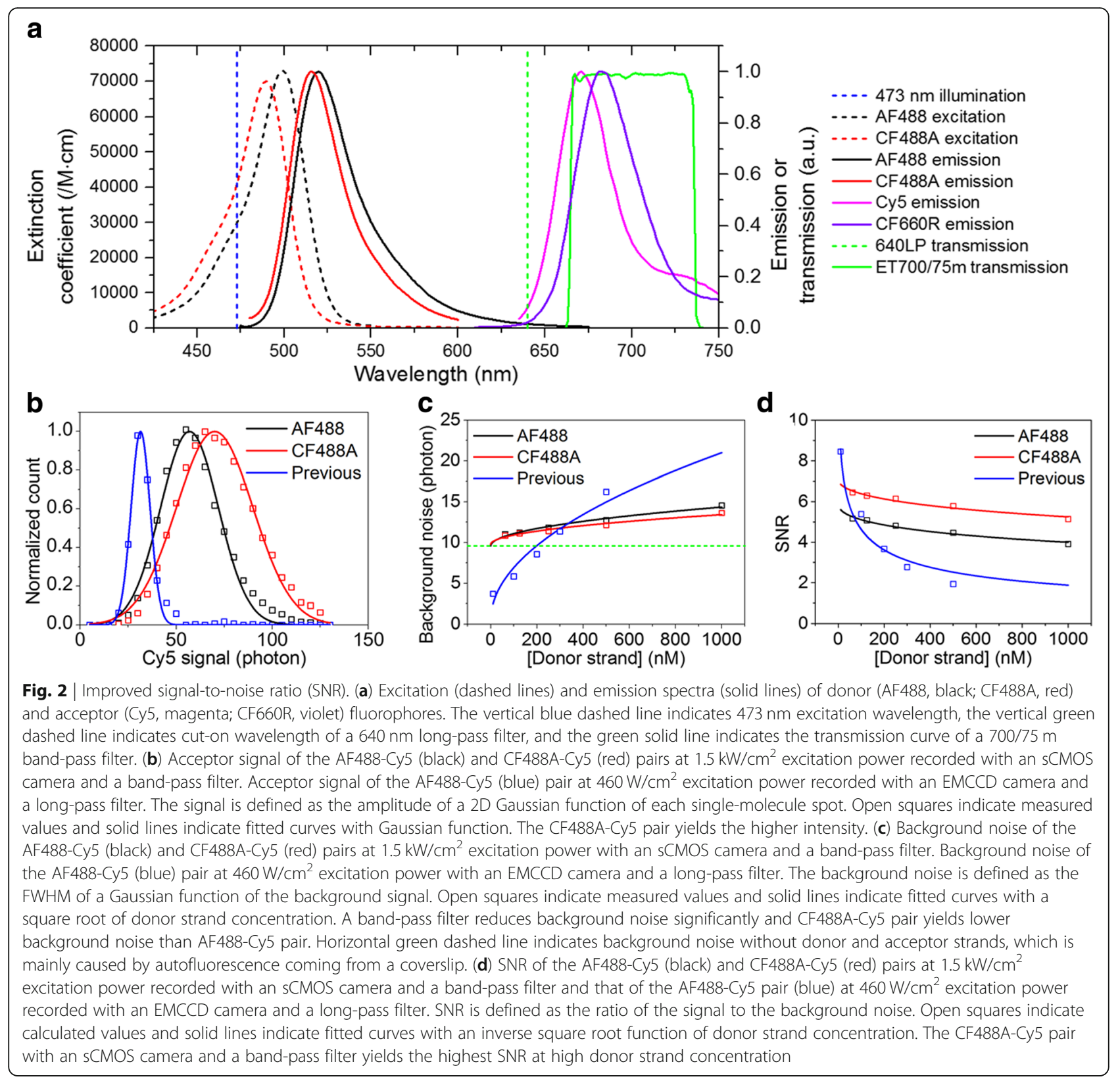

effort to improve SNR, we also replaced a $640 \mathrm{~nm}$ long-pass filter (green dashed line) with a 700/75 band-pass filter (green solid line). Since the band-pass filter has a red-shifted cut-on wavelength than the long-pass filter, some portion of acceptor signal is lost by the replacement, but we expected the reduction of donor bleed-through would increase SNR at high donor strand concentrations. As expected from the fact that CF488A has larger extinction coefficient than AF488 at $473 \mathrm{~nm}$, the CF488A-Cy5 pair gave more photons than the AF488-Cy5 pair at the same excitation power (Fig. 2b). The background noise, and thus the signal to noise ratio, were improved dramatically by using a band-pass filter instead of a long-pass filter. And the background noise and the signal to noise ratio were also improved with the CF488A-Cy5 pair than the AF488-Cy5 pair (Fig. 2c, d). It is noticeable that the optimization process mentioned above removed the donor bleed-through almost completely, and as a result, the dependence of SNR on donor concentration was very weak (Fig. 2d). Contrary to our expectation, we found that replacement of Cy5 with CF660R did not improve SNR because CF660R has higher direct excitation than Cy5 at $473 \mathrm{~nm}$ (Additional file 1: Figure S1). Since CF660R has lower direct excitation than $\mathrm{Cy} 5$ at $488 \mathrm{~nm}$, we expect that CF660R may provide better performance if we use a 488-nm excitation laser instead of the 473-nm laser in a future work. In this work, we exclusively used the CF488A-Cy5 pair at $473 \mathrm{~nm}$ excitation. 
Characterization of the imaging speed of a new microscope To characterize the improved imaging speed of the new microscope, we compared the imaging speed of the new FRET-PAINT microscope with the previous one. As a model system, microtubules of COS-7 cells were imaged. $7 \mathrm{nt}$ donor strands and $1.5 \mathrm{~kW} / \mathrm{cm}^{2}$ excitation power were used for the new microscope whereas $9 \mathrm{nt}$ donor strands and $460 \mathrm{~W} / \mathrm{cm}^{2}$ excitation power were used for the old one. Figure 3a shows a super-resolution image obtained with the old microscope using a $10 \mathrm{~Hz}$ frame rate and $1 \mathrm{~min}$ acquisition time. For the imaging, $30 \mathrm{nM}$ AF488-labeled donor strands and $20 \mathrm{nM}$ Cy5-labeled acceptor strands were used. Figure $3 \mathrm{~b}$ and $\mathrm{c}$ show super-resolution images obtained with the new microscope using $100 \mathrm{~Hz}$ frame rate for Fig. 3b or $200 \mathrm{~Hz}$ for Fig. 3c. For the imaging, the total data acquisition time was $1 \mathrm{~min}$, and $300 \mathrm{nM}$ CF488A-labeled donor strands and $300 \mathrm{nM}$ Cy5-labeled acceptor strands were used. As clear from the figures, the new microscope provided higher quality images than the previous FRET-PAINT setup in a shorter time. The cross-sectional width of microtubules was similar to the previously reported value (Additional file 1: Figure S2) [11]. To show the improved image qualities in more detail, time-lapse images of the boxed regions of Fig. 3a-c are also shown in Fig. 3d-f, respectively. To quantitatively compare image qualities of Fig. 3a-c, we compared the image resolutions as a function of image acquisition time (Fig. 3g). The resolutions were obtained using the Fourier ring correlation method $[16,17]$. It is noticeable that the resolution arrived at the limit ( $42 \mathrm{~nm}$ for $100 \mathrm{~Hz}, 46 \mathrm{~nm}$ for $200 \mathrm{~Hz}$ ) after $20-30 \mathrm{~s}$ with the new FRET-PAINT setup whereas the resolution still decreases even after $60 \mathrm{~s}$ with the previous FRET-PAINT setup. In principle, the resolution defined by Fourier ring correlation method is affected by both the localization precision and the localization density [16-19]. The localization density is linearly proportional to the imaging time (Fig. 3h) whereas the localization precision is time-independent. Therefore we can conclude that for tens of seconds imaging time the image resolution is determined by the localization precision in a new microscope. For the same image acquisition time, on the other hand, the image resolution is determined by the localization density in the old microscope. Localization density as a function of imaging time in Fig. 3h provides another way to compare the imaging speed of the microscopes. The localization rate was increased by 5.4 times for the $100 \mathrm{~Hz}$ imaging, and 8 times for the $200 \mathrm{~Hz}$ imaging.

\section{Discussion}

In summary, we developed a high-speed FRET-PAINT microscope that can provide localization-precision limited super-resolution images in tens of seconds. For the achievement, we optimized several experimental parameters such as the camera speed, dissociation time of donor strands, and bleed-through of donor signals to the acceptor channel.

Compared to the work of Auer et al. who claimed that super-resolution images of microtubules could be obtained in a few tens of seconds [11], our work has several improvements in the following respects. First, they used a docking strand permanently-labeled with an acceptor for cell imaging, and as a result the total imaging time of their approach was still limited by photobleaching of the acceptor. Second, they used 9 nt donor strand whose dissociation time is estimated to be around $1 \mathrm{~s}$. Even though they used high donor concentration (500 $\mathrm{nM})$, and short integration time (14 ms), single-molecule spots seriously overlap with $9 \mathrm{nt}$ donor strand, and as a result, overall imaging speed of their approach is determined by the donor dissociation time as fully demonstrated in our previous paper [10]. It is not possible to directly compare imaging speed of their approach with ours because Auer et al. characterized the localization precision only using a nearest neighbor analysis [20] without information about the localization density for image reconstruction; they provided potentially achievable spatial resolution only, but not the experimentally achieved actual resolution. However, we think that their imaging speed was similar to that of our previous version of a FRET-PAINT microscope where we used $9 \mathrm{nt}$ donor strand. The resolution of super-resolution fluorescence microscopy based on single-molecule localization is not solely determined by the localization precision of single-molecules but the localization density of single-molecules for image reconstruction should be also considered. Therefore, the increased imaging speed in the work compared to the previous works $[10,11]$ means improved resolution for the same imaging time (Fig. 3g).

Then, have we arrived at the ultimate speed limit of FRET-PAINT microscopy? We believe that there still is a room for improvements. Figure $2 d$ shows that we could use much higher donor strand concentrations than $300 \mathrm{nM}$ without compromising SNR. The localization precision could be also improved by collecting more photons. By using 6 nt donor stand, the donor strand switching rate could be also increased. Incorporation of all these change into the microscope to increase the imaging speed, however, requires higher excitation intensity to compensate for the decreased photon number caused by the reduced binding lifetime of the probes. Unfortunately, we found that this simple scheme did not work; we found that the number of single-molecule spots decreased in a laser-power dependent fashion as imaging went on (Additional file 1: Figure S3a). Therefore, we concluded that DNA probes used in FRET-PAINT were damaged by the high-intensity excitation laser. This kind of photo-induced damage was not 

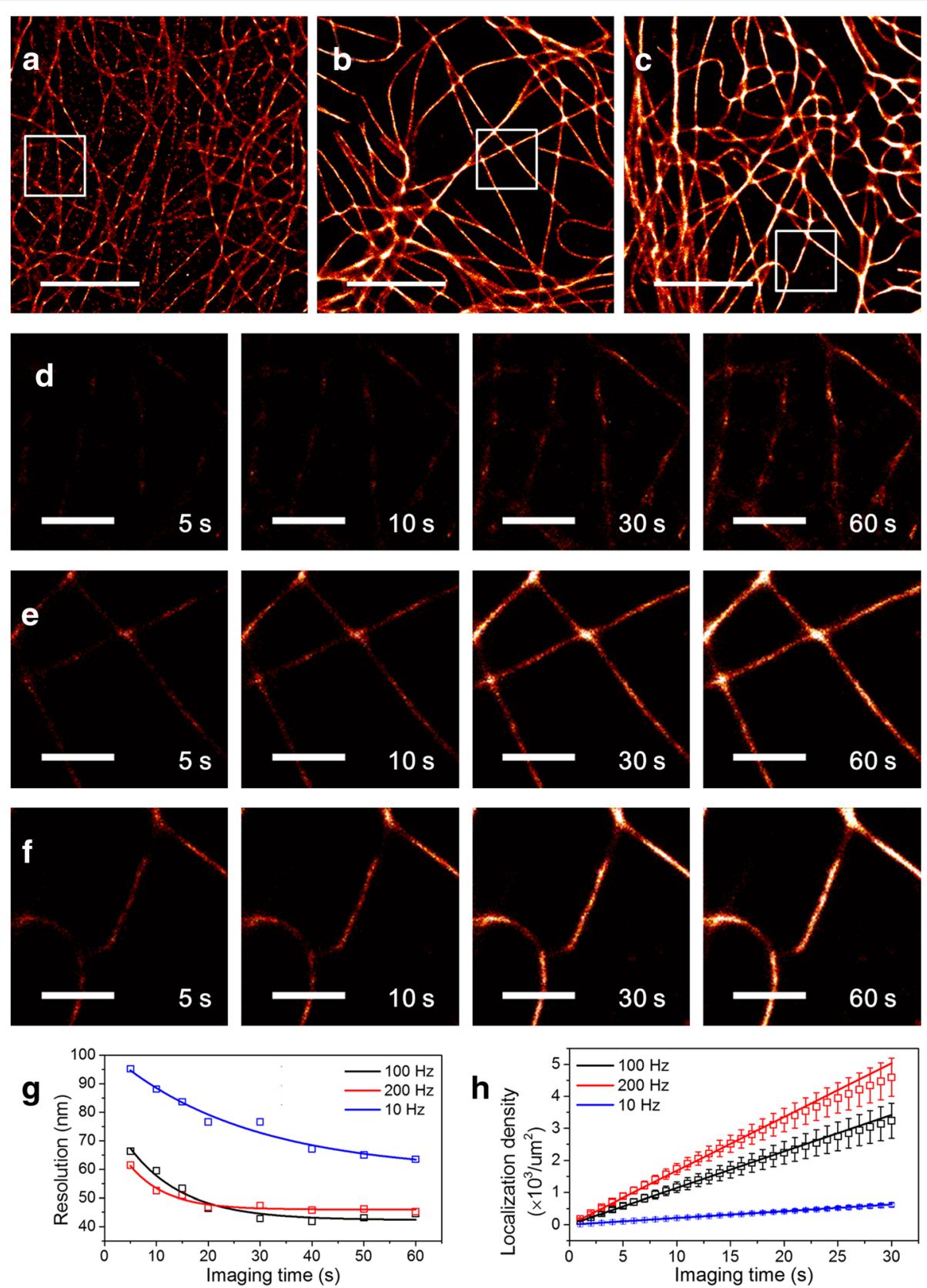

Fig. 3 Characterization of the imaging speed of a new microscope. Super-resolution microtubule images of fixed COS-7 cells were used as a model system. (a) The image was reconstructed from 600 frames recorded at a frame rate of $10 \mathrm{~Hz}$ with a previous microscope (an EMCCD camera, a long-pass filter, $460 \mathrm{~W} / \mathrm{cm}^{2}$ excitation power, $30 \mathrm{nM} 9 \mathrm{nt}$ AF488 donor strands, $20 \mathrm{nM} 10 \mathrm{nt}$ Cy5 acceptor strands). (b, c) The images were reconstructed from 6000 frames recorded at a frame rate of $100 \mathrm{~Hz}(\mathbf{b})$ or 12,000 frames recorded at a frame rate of $200 \mathrm{~Hz}$ (c) with a new microscope (an sCMOS camera, a band-pass filter, $460 \mathrm{~W} / \mathrm{cm}^{2}$ excitation power, $300 \mathrm{nM} 7 \mathrm{nt}$ CF488A donor strands, $300 \mathrm{nM} 9 \mathrm{nt}$ Cy5 acceptor strands). An imaging buffer (10 mM Tris-HCl, pH 8.0, $500 \mathrm{mM} \mathrm{NaCl}, 1 \mathrm{mg} / \mathrm{ml}$ glucose oxidase, $5 \mathrm{mg} / \mathrm{ml}$ glucose, $0.04 \mathrm{mg} / \mathrm{ml}$ catalase, and $1 \mathrm{mM}$ Trolox) was used for all imaging. All images were reconstructed using ThunderSTORM [23] with maximum likelihood fitting method. Total imaging time is $60 \mathrm{~s}$ for all images. (d-f) Time-lapse images of the boxed regions in a-c at the specified imaging time. (g) Image resolutions of a-c using Fourier ring correlation method as a function of the imaging time. Open squares indicate measured value and solid lines indicate fitted curves with an exponential decay function. (h) A localization density as a function of the imaging time (100 Hz, black; $200 \mathrm{~Hz}$, red; $10 \mathrm{~Hz}$, blue). The localization density is defined as the number of localization events per um $^{2}$. To minimize the influence of the region of interest selected for data analysis, the localization density was calculated from 10 different regions of 5 different cells. Squared boxes indicate the average and error bars indicate the standard deviation. The increase rates of the localization density were 21 (10 Hz), $114(100 \mathrm{~Hz})$, and 168 (200 Hz) localizations/ $\mathrm{um}^{2} / \mathrm{s}$. We obtained 5.4 times increase for $100 \mathrm{~Hz}$ imaging, and 8 times increase for $200 \mathrm{~Hz}$ imaging compared to the old microscope. Scale bars: 5 um (a-c), 1 um (d-f) 
recognized in the previous works where relatively weak excitation power was used [10,11]. To pin down what kind of damage occurred, we performed additional experiments. The injection of fresh DNA probes did not solve the problem (Additional file 1: Figure S3b). The background noise of fluorophores did not decrease during imaging (Additional file 1: Figure S3c). Therefore, the damage is not simple photobleaching of fluorophores but seems to be the loss of base-pairing capability of the docking strand. Interestingly, we found that the photo-induced damage exhibited sample-to-sample variation (Additional file 1: Figure S3d). Finding of a way to systematically solve the photo-induced problem will enable us to realize sub-millisecond image acquisition for super-resolution imaging. When combined with a recently-developed real-time confocal microscopy [21, 22], our accelerated FRET-PAINT microscopy will provide a way to reconstruct three-dimensional structures of thick neural tissue samples with both high speed and high resolution.

\section{Methods}

\section{Materials}

Modified DNA oligonucleotides were purchased from Bioneer (Daejeon, Republic of Korea). AF488 (Alexa Fluor 488 NHS Ester, catalog number: A20000) and Nunc Lab-Tek chambered coverglass (catalog number: 155383PK) were purchased from Thermo Fisher Scientific. CF488A ( $\mathrm{CF}^{\circ} 488 \mathrm{~A}$ Succinimidyl Ester, catalog number: 92120) and CF660R (CF $660 \mathrm{R}$ Succinimidyl Ester, catalog number: 92134) were purchased from Biotium. Cy5 (Cy5 NHS Ester, catalog number: PA15101) was purchased from GE Healthcare Life Sciences. COS-7 cells were purchased from Korean Cell Line Bank. Anti-tubulin antibody (catalog number: ab6160) was purchased from Abcam. Donkey anti-rat IgG antibody (catalog number: 712-005-153) was purchased from Jackson ImmunoResearch Laboratories, Inc. The docking strands were conjugated to the secondary antibodies using Antibody-Oligonucleotide All-in-One Conjugation Kit (catalog number: A-9202-001) purchased from Solulink. Glutaraldehyde (catalog number: G5882), Triton X-100 (catalog number: T9284), Sodium Borohydride (catalog number: 452882-5G), and Bovine Serum Albumin (catalog number: A4919) were purchased from Sigma-Aldrich.

\section{DNA labeling with fluorophores}

Amine-modified DNA oligonucleotides were labeled with fluorophores which have an NHS ester chemical group. $5 \mathrm{ul}$ of $1 \mathrm{mM}$ DNA was mixed with $25 \mathrm{ul}$ of 100 $\mathrm{mM}$ sodium tetraborate buffer ( $\mathrm{pH} 8.5$ ). And then $5 \mathrm{ul}$ of $20 \mathrm{mM}$ fluorophore in DMSO was added. After thorough mixing, the mixture was incubated at $4{ }^{\circ} \mathrm{C}$ overnight while protected from light. 265 ul of distilled water, $900 \mathrm{ul}$ of ethanol, and $30 \mathrm{ul}$ of $3 \mathrm{M}$ sodium acetate (pH 5.2) were added and mixed thoroughly. The mixture was incubated at $-20^{\circ} \mathrm{C}$ for an hour and then centrifuged for a couple of hours until the DNA pellet is clearly visible. If the pellet is not visible, the mixture was incubated at $-20^{\circ} \mathrm{C}$ overnight or several days. The supernatant was discarded and the pellet was washed with cold ethanol. After ethanol was evaporated completely, the pellet was resuspended in $50 \mathrm{ul}$ of MilliQ water and the labeling efficiency was measured with a spectrophotometer (Nanodrop 2000, Thermo Fisher Scientific). If the labeling efficiency is low, the whole labeling process was repeated. If the labeling efficiency exceeds $100 \%$, the purification step was repeated.

\section{Cell culture, fixation, and immunostaining}

COS-7 cells were grown on Nunc Lab-Tek chambered coverglass for a day. The cells were briefly washed twice with $37^{\circ} \mathrm{C}$ PBS buffer, pre-extracted with $37^{\circ} \mathrm{C}$ pre-extraction buffer $(0.4 \%$ glutaraldehyde, $0.25 \%$ Triton X-100 in PBS buffer) for $20 \mathrm{~s}$, fixed with $37^{\circ} \mathrm{C}$ fixation buffer (3\% glutaraldehyde in PBS buffer) for $10 \mathrm{~min}$, and washed with PBS buffer 3 times (5 min each) to remove unreacted free glutaraldehyde molecules. Unreacted aldehyde groups were quenched with quenching buffer $(1 \mathrm{mg} / \mathrm{ml}$ sodium borohydride in PBS buffer) 3 times (4 min each). And then, the cells were washed with PBS buffer 3 times ( $5 \mathrm{~min}$ each). Microtubules were immunostained by injecting 1:100 diluted primary anti-tubulin antibodies in blocking buffer $(5 \%$ Bovine Serum Albumin and $0.25 \%$ Triton X-100 in PBS buffer) into the chamber and incubating at room temperature for an hour. Free anti-tubulin antibodies were washed with blocking solution 3 times ( $5 \mathrm{~min}$ each). And then, $100 \mathrm{nM}$ secondary antibodies conjugated with docking strands were injected into the chamber and incubated at room temperature for an hour. Free secondary antibodies were washed with the PBS buffer 3 times (5 min each).

\section{Single-molecule fluorescence imaging}

For FRET-PAINT imaging, we used $7 \mathrm{nt}$ CF488A-labeled donor strand, and 9 nt Cy5-labeled acceptor strand. Excitation power was $1.5 \mathrm{~kW} / \mathrm{cm}^{2}$ at $473 \mathrm{~nm}$. Cy5 signal was filtered with a band-pass filter (ET700/75 m, Chroma), and imaged using an inverted microscope (IX71, Olympus) equipped with an oil-immersion objective $(100 \times 1.4$ NA, UPlansSApo, Olympus) and an sCMOS camera (ORCA-Flash 4.0 V2, Hamamatsu) with 5 or $10 \mathrm{~ms}$ integration time. For all experiments, an identical imaging buffer $(10 \mathrm{mM}$ Tris- $\mathrm{HCl}, \mathrm{pH} 8.0,500 \mathrm{mM} \mathrm{NaCl}, 1 \mathrm{mg} / \mathrm{ml}$ glucose oxidase, $5 \mathrm{mg} / \mathrm{ml}$ glucose, $0.04 \mathrm{mg} / \mathrm{ml}$ catalase in saturated Trolox solution) was used. 


\section{Additional file}

Additional file 1: Accelerated FRET-PAINT Microscopy. Figure S1. Excitation spectra of Cy5 (black) and CF660R (red). Figure S2. A crosssectional histogram of microtubules. Figure S3. Photo-induced damage of DNA probes. (DOCX $538 \mathrm{~kb}$ )

\section{Abbreviations}

AF488: Alexa Fluor 488; PAINT: Point Accumulation for Imaging in Nanoscale Topography; SNR: Signal-to-Noise Ratio

\section{Acknowledgements}

Not applicable.

\section{Funding}

This work was supported by a Creative Research Initiative grant (Physical Genetics Laboratory, 2009-0081562) to S.H.

\section{Availability of data and materials}

The datasets generated and analyzed during the current study are available from the corresponding author on reasonable request.

\section{Authors' contributions}

$J$ and SH designed experiments. $J$ and SP performed experiments and analyzed data. All authors contributed to writing of the paper. S.H. supervised the research. All authors read and approved the final manuscript.

Ethics approval and consent to participate

Not applicable.

\section{Consent for publication}

Not applicable.

\section{Competing interests}

The authors declare that they have no competing interests.

\section{Publisher's Note}

Springer Nature remains neutral with regard to jurisdictional claims in published maps and institutional affiliations.

Received: 7 August 2018 Accepted: 9 November 2018

Published online: 22 November 2018

\section{References}

1. Hell SW, Wichmann J. Breaking the diffraction resolution limit by stimulated emission: stimulated-emission-depletion fluorescence microscopy. J Opt Lett. 1994;19:780-2.

2. Hell SW, Kroug M. Ground-state-depletion fluorscence microscopy: a concept for breaking the diffraction resolution limit. Appl Phys B Lasers Opt. 1995:60:495-7.

3. Gustafsson MGL. Surpassing the lateral resolution limit by a factor of two using structured illumination microscopy. J Microsc. 2000;198:82-7.

4. Gustafsson MGL. Nonlinear structured-illumination microscopy: wide-field fluorescence imaging with theoretically unlimited resolution. Proc Natl Acad Sci U S A. 2005;102:13081-6.

5. Betzig E, Patterson GH, Sougrat R, Lindwasser OW, Olenych S, Bonifacino JS, Davidson MW, Lippincott-Schwartz J, Hess HF. Imaging intracellular fluorescent proteins at nanometer resolution. Science. 2006;313:1642-5.

6. Hess ST, Girirajan TPK, Mason MD. Ultra-high resolution imaging by fluorescence Photoactivation localization microscopy. Biophys J. 2006;91: 4258-72.

7. Rust MJ, Bates M, Zhuang X. Sub-diffraction-limit imaging by stochastic optical reconstruction microscopy (STORM). Nat Methods. 2006;3:793-6.

8. Sharonov A, Hochstrasser RM. Wide-field subdiffraction imaging by accumulated binding of diffusing probes. Proc Natl Acad Sci U S A. 2006; 103:18911-6.

9. Jungmann $R$, Steinhauer $C$, Scheible M, Kuzyk A, Tinnefeld P, Simmel FC. Single-molecule kinetics and super-resolution microscopy by fluorescence imaging of transient binding on DNA origami. Nano Lett. 2010;10:4756-61.
10. Lee J, Park S, Kang W, Hohng S. Accelerated super-resolution imaging with FRET-PAINT. Mol Brain. 2017;10:63.

11. Auer A, Strauss MT, Schlichthaerle T, Jungmann R. Fast, background-free DNA-PAINT imaging using FRET-based probes. Nano Lett. 2017;17:6428-34.

12. Spectra Viewer. https://www.thermofisher.com/order/spectra-viewer. Accessed 2 Oct 2017

13. Absorbance Spectra. http://www.atto-tec.com/index.php?id=256\&no_ cache $=1 \& L=1$. Accessed 2 Oct 2017.

14. Emission Spectra. http://www.atto-tec.com/index.php?id=258\&no_cache= $1 \& L=1$. Accessed 2 Oct 2017

15. Absorption and Emission Normalized Data. https://biotium.com/wpcontent/uploads/2016/03/CF-Dyes-Absorption-and-Emission-NormalizedData-web.xls. Accessed 2 Oct 2017.

16. Banterle N, Bui KH, Lemke EA, Beck M. Fourier ring correlation as a resolution criterion for super-resolution microscopy. J Struct Biol. 2013;183:363-7.

17. Nieuwenhuizen RPJ, Lidke KA, Bates M, Puig DL, Grünwald D, Stallinga S, Rieger B. Measuring image resolution in optical nanoscopy. Nat Methods. 2013;10:557-62.

18. Jones SA, Shim SH, He J, Zhuang X. Fast, three-dimensional super-resolution imaging of live cells. Nat Methods. 2011;8:499-508.

19. Shroff H, Galbraith CG, Galbraith JA, Betzig E. Live-cell photoactivated localization microscopy of nanoscale adhesion dynamics. Nat Methods. 2008:5:417-23.

20. Endesfelder U, Malkusch S, Fricke F, Heilemann M. A simple method to estimate the average localization precision of a single-molecule localization microscopy experiment. Histochem Cell Biol. 2014;141:629-38.

21. Lee J, Miyanaga Y, Ueda M, Hohng S. Video-rate confocal microscopy for single-molecule imaging in live cells and superresolution fluorescence imaging. Biophys J. 2012;103:1691-7.

22. Park S, Kang W, Kwon YD, Shim J, Kim S, Kaang BK, Hohng S. Superresolution fluorescence microscopy for 3D reconstruction of thick samples. Mol. Brain. 2018;11:17.

23. Ovesný M, Kř́žžek P, Borkovec J, Švindrych Z, Hagen GM. ThunderSTORM: a comprehensive ImageJ plug-in for PALM and STORM data analysis and super-resolution imaging. Bioinformatics. 2014;30:2389-90.

\section{Ready to submit your research? Choose BMC and benefit from:}

- fast, convenient online submission

- thorough peer review by experienced researchers in your field

- rapid publication on acceptance

- support for research data, including large and complex data types

- gold Open Access which fosters wider collaboration and increased citations

- maximum visibility for your research: over $100 \mathrm{M}$ website views per year

At $\mathrm{BMC}$, research is always in progress.

Learn more biomedcentral.com/submissions 